\title{
Reflection and refraction of acoustic waves at poroelastic ocean bed
}

\author{
A. K. Vashishth and M. D. Sharma \\ Dept. of Mathematics, Kurukshetra University, India-136119 \\ (Received December 27, 2007; Revised October 6, 2008; Accepted November 27, 2008; Online published July 27, 2009)
}

\begin{abstract}
Ocean bottom is considered as a plane interface between non-viscous liquid and anisotropic dissipative poroelastic solid. The dissipation comes from the viscosity of pore-fluid as well as the anelasticity of the porous frame. Biot's theory is used to derive a system of modified Christoffel equations for the propagation of plane harmonic waves in a porous medium. The non-trivial solution of this system is ensured by a determinantal equation. This equation is solved into a polynomial equation of degree eight, whose roots represent the vertical slowness values for the waves propagating upward and downward in a porous medium. The eight, numerically obtained, slowness values are identified with the four waves propagating towards (or away from) the boundary in the porous medium. Incidence of acoustic wave through the liquid at the interface results in its reflection and the refraction of four attenuating waves in the poroelastic medium. The energy partition among the reflected and refracted waves is calculated at the interface. Conservation of energy is obtained except in the case of partially opened surface pores of the porous medium. Energy refracted to the dissipative porous medium is expressed through an energy matrix. The four diagonal elements of this matrix represent the energy shares of the four inhomogeneous waves and the sum of all the off-diagonal elements of this matrix represents the interaction energy. Few significant results are extracted from the observations in the numerical examples studied. These results represent the effect of anisotropic symmetries, anelasticity, wave-frequency, opening, configuration and flow-impedance of pores, on the energy shares of reflected and refracted waves.
\end{abstract}

Key words: Poroelasticity, anisotropy, ocean-bottom, reflection, refraction, acoustic.

\section{Introduction}

The enhanced recovery of hydrocarbons, triggering of earthquakes, underwater acoustics and non-destructive evaluation of composite materials are some of the important areas which demand more attention to the theoretical studies of wave propagation in fluid-saturated porous medium. Ocean covers nearly three-fourth of earth's surface and sediments at ocean-bottom provide the most appropriate examples of saturated porous materials in the crust.

The dynamic theory for wave propagation in fluidsaturated porous media was developed by Biot (1956, 1962a, b). Most of the studies, carried thereafter, on wave propagation in porous media are based on Biot's theories. Stoll and Bryan (1970) described a mathematical model for ocean sediments which was based on the classical work of Biot. Stoll $(1974,1977,1979,1980)$ studied wave propagation in ocean sediments considering both theoretical aspects and experimental studies on transmission and attenuation of acoustic waves in sediments. Reflection and refraction of plane acoustic waves at water-sediment interface were studied by Stoll and Kan (1981) where sediments were modeled as a porous viscoelastic medium. The acoustic normal modes in an ocean overlying a poroelastic bottom half-space were studied by Yamamoto (1983). Kohler (1988) studied the structure of the fields within sediments based on a one-dimensional randomly stratified model of

Copyright (c) The Society of Geomagnetism and Earth, Planetary and Space Sciences (SGEPSS); The Seismological Society of Japan; The Volcanological Society of Japan; The Geodetic Society of Japan; The Japanese Society for Planetary Sciences; TERRAPUB. ocean sediments. Kuo (1992) calculated reflection loss during acoustic wave scattering from solid boundaries at the ocean bottom. de la Cruz et al. (1992) analyzed the mode conversions which occur during the reflection and transmission of seismic waves at the boundaries of porous media. Collins et al. (1997) found that some ocean sediments may be modeled as poroelastic media with relatively high speed of slow-dilatational wave and relatively low speed of shear wave. Buckingham $(1997,1998,1999,2000)$ studied the wave propagation for wave speeds and attenuations in consolidated and non-consolidated marine sediments. The effects of acoustic properties of seabed, including the density and sound speed of the sedimentary layer and sub-bottom, on the wave-field characteristics were investigated by Liu et al. (2001). Surface displacements, surface strain, rocking and energy partitioning during the reflection of plane waves in a fluid-saturated poroelastic half-space were discussed by Lin et al. (2006). They inferred that amount of solid frame stiffness controls the response of a fluid-saturated porous system. Sound penetration from acoustic wide-band array buried into a sandy sediment was observed by Chotiras et al. (2007) to study the refraction and scattering into sandy ocean sediments.

The mechanics of a ocean sediment is complicated because it is a coupled multiphase medium consisting of a solid phase of grains and fluid phases of pore water and gases. Porosity, gradation and grain shape, material properties of individual grains, static intergranular stress, dynamic strain amplitude, degree and kind of lithification and structure as determined by the mode of deposition are some 
of the important parameters which affect the dynamic response of saturated sediments (Stoll, 1980). The preferential alignments in the Earth ranging from mineral orientations, grain, or micro cracks to regional fractures result in the seismic anisotropy (Crampin, 1994). According to spectral analysis of many cross-well acoustic tomograms and cores collected from seabed locations, the 3-D power spectra of velocity and density fluctuations in the seabed sediments are strongly anisotropic and dipping in general (Yamamoto, 1996). Gurevich (2003) investigated that elastic properties of fluid-saturated porous rocks with aligned fractures can be studied using the model of fractures as linear slip interfaces in an isotropic porous background. It was further concluded that such a medium can be represented as anisotropic poroelastic aggregate. In water-saturated particulate material such as ocean sediments, energy loss occurs due to friction and/or anelasticity of any bond that exists at the points of intergranular contact and due to the viscous losses when there is any relative motion between the fluid and the skeletal frame. The mechanics of ocean sediment becomes further complicated when anisotropy and anelasticity are considered.

Based on Biot's theory, some problems on wave propagation in anisotropic fluid-saturated porous solid have also been studied. Schmitt (1989) investigated the properties of the modes generated by multipole sources in a fluidfilled borehole embedded in foundation that include transversely isotropic poroelastic layers. Sharma and Gogna (1991) studied Rayleigh waves on the surface of transversely isotropic liquid-saturated porous layered medium. Potel and de Belleval (1993) discussed wave propagation in an anisotropic periodically multilayered medium. Badiey et al. (1994) used the propagator matrix method to study plane wave reflection from inhomogeneous anisotropic poroelastic seafloor. Vashishth and Khurana (2005) studied Rayleigh modes in anisotropic heterogeneous poroelastic layers. The anisotropy, considered in these studies, was of simple type, e.g., transverse isotropy. Recently, Sharma (2004a, 2005) established three-dimensional mathematical models for propagation of four waves in general anisotropic fluid-saturated porous solids. Using the Biot's theory of 1956, Sharma (2004b) studied the reflection and refraction at a plane interface between fluid and a non-dissipative porous solid. The transmitted waves in the non-dissipative porous medium were assumed to be homogeneous waves.

In this paper, we study the reflection and transmission of plane waves at a plane interface between water and anisotropic poro-viscoelastic solid, which is a realistic model of ocean sediments. The elastic/hydraulic anisotropy of arbitrary type, anelastic porous frame, viscous pore-fluid, partially opened surface pores, impedance to pore-fluid discharge are major attributes of the medium considered to model ocean sediments. Energy losses, both in skeletal frame of the sediment and the viscous losses in the pore liquid as it moves relative to the frame, are taken into account keeping into consideration the two-component nature of porous materials. Purpose of the study is to explore the role of these geophysical attributes on the partition of incident acoustic energy among reflected acoustic wave and the waves refracted to porous bed. Numerical examples are considered to analyse the effects of geophysical properties of the porous bed on the variations of energy shares of different waves with the direction of incident acoustic wave. Conservation of energy is verified/analysed in all the examples.

\section{Anisotropic Poroelastic Propagation}

Following Biot (1962a, b), a set of differential equations governs the particle motion in a general anisotropic fluidsaturated porous solid. These equations, in the absence of body forces, are given by

$$
\begin{gathered}
c_{i j k l} u_{k, j l}+m_{i j} w_{k, j k}=\rho \ddot{u}_{i}+\rho_{\mathrm{f}} \ddot{w}_{i}, \\
m_{k j} u_{k, i j}+R w_{k, i k}=\rho_{\mathrm{f}} \ddot{u}_{i}+q_{i j}^{\prime} \ddot{w}_{j},
\end{gathered}
$$

where, $c_{i j k l}$ are the elastic constants of the porous aggregate. Another elastic constant $R$ measures the pressure to be exerted on the fluid to push its unit volume into the porous matrix. The parameters $m_{i j}$ and $q_{i j}^{\prime}$ control the elastic and inertial coupling between fluid and solid phases of the porous aggregate. The $\rho$ and $\rho_{\mathrm{f}}$ are densities of porous aggregate and pore-fluid, respectively. $u_{i}$ are the components of the average displacements for the solid and $w_{i}$ are the components of displacement of fluid relative to the solid. A repeated (dummy) index implies summation, dot represents (partial) time derivative and comma before an index denotes partial space differentiation.

The complex values of $c_{i j k l}, R, m_{i j}$ represent the anelastic character of the porous frame whereas the complex values of $q_{i j}^{\prime}$ define the dissipation due to pore-fluid viscosity. The effect of viscosity $(\mu)$ of pore fluid is restricted only to dissipation due to the relative motion between fluid and solid particles. Following Johnson et al. (1987) and Albert (1993), $q_{i j}^{\prime}$ can be written in terms of anisotropic permeability $\left(\kappa_{i j}\right)$ of solid matrix and tortuosity of pores $\left(\alpha_{\infty}\right)$ for non-viscous pore-fluid as

$$
q_{i j}^{\prime}=\rho_{\mathrm{f}} \frac{\alpha_{\infty}}{f}\left(\delta_{i j}+d_{i j}\right),
$$

where $\delta_{i j}$ is Kronecker delta. Writing the permeability tensor $\kappa\left(=\kappa_{\mathrm{o}} \kappa_{\mathrm{a}}\right)$, the dissipation matrix $\mathbf{d}\left\{d_{i j}\right\}$ is defined as

$$
\mathbf{d}=\imath \frac{1}{\Omega}\left\{\kappa_{\mathrm{a}}\right\}^{-1} ; \quad \Omega=\omega / \omega_{\mathrm{c}} ; \quad \omega_{\mathrm{c}}=\frac{\mu}{\rho_{\mathrm{f}} \Delta},
$$

where, $\Delta\left(=\kappa_{\mathrm{o}} \alpha_{\infty} / f\right)$ is a parameter with dimension of permeability. $\omega$ is the circular frequency of plane harmonic wave with $e^{-l \omega t}$ time dependence. The expression of $\mathbf{d}$, in (3), is valid for low frequency, when the flow in pores is of Poiseuille type. For high frequency, the viscosity $\mu$ is multiplied by a frequency dependent correction factor

$$
F(\omega)=\left[1-\imath \frac{1}{2} \eta \Omega\right]^{1 / 2} ; \quad \eta=8 \frac{\Delta}{\Lambda^{2}},
$$

where, the viscous characteristic length $\Lambda$ represents the configuration of pores. The value of non-dimensional parameter $\eta$ is around 1 , for most of the porous media. Hence, the dissipation $\mathbf{d}$ in the medium becomes an explicit function of two parameters $\Omega$ and $\eta$. 
For the propagation of plane waves with $e^{-l \omega t}$ time dependence, consider the harmonic solution

$$
\begin{aligned}
u_{j} & =S_{j} \exp \left\{i \omega\left(p_{k} x_{k}-t\right)\right\} ; \\
w_{j} & =F_{j} \exp \left\{i \omega\left(p_{k} x_{k}-t\right)\right\},
\end{aligned}
$$

where $\left(p_{1}, p_{2}, p_{3}\right)$ is the slowness vector. The vector $\left(S_{1}, S_{2}, S_{3}\right)$ represents the normalised polarisation of solid particles and $\left(F_{1}, F_{2}, F_{3}\right)$ is the polarisation of the fluid motion relative to the solid matrix.

Substitution of (5) in (1) yields a system of six homogeneous equations, which is manipulated further (Sharma, 2005) to obtain two subsystems. One of these relates the displacements of fluid and solid particles by a relation

$$
w_{i}=\Gamma_{i k} u_{k} ; \quad \Gamma=\frac{1}{R}\left[\boldsymbol{\chi}-\mathbf{p}^{T} \mathbf{p}\right]^{-1}\left[\mathbf{p}^{T} \mathbf{p} \mathbf{M}-\rho_{\mathrm{f}} \mathbf{I}\right],
$$

where, matrix $\boldsymbol{\chi}=\frac{1}{R}\left\{\mathbf{q}^{\prime}\right\}, \mathbf{M}=\left\{m_{i j}\right\}$ and $\mathbf{I}$ is identity matrix of order three. The slowness vector $\mathbf{p}$ is considered a row-matrix and $\mathbf{p}^{\mathrm{T}}$ is its transpose. The other system defines the Christoffel equations for the motion of solid particles, and, is given by

$$
\begin{aligned}
& \mathbf{W} \mathbf{S}=\mathbf{0} ; \\
& W_{i k}=c_{i j k l} p_{j} p_{l}-\rho \delta_{i k}+\left(m_{i l} p_{j} p_{l}-\rho_{\mathrm{f}} \delta_{i j}\right) \Gamma_{j k} .
\end{aligned}
$$

The non-trivial solution of the Christoffel equations is ensured by a determinantal equation, given by

$$
\operatorname{det} \mathbf{W}=0 .
$$

To solve this equation, assume that $p_{1}, p_{2}$ are known and an unknown $q$ denotes the vertical slowness $p_{3}$. Then, in terms of $q$, the matrix $\Gamma$ in (6) is given by

$$
\Gamma=\left[\Gamma^{(2)} q^{2}+\Gamma^{(1)} q+\Gamma^{(0)}\right] /\left[R\left(a_{2} q^{2}+a_{1} q+a_{0}\right)\right],
$$

where, for $X_{I J}=\chi_{I I} \chi_{J J}-\chi_{I J}^{2}, \quad X_{I J K}=\chi_{I J} \chi_{J K}-$ $\chi_{I K} \chi_{J J} ; \quad I \neq J \neq K=1,2,3$; (no summation over repeated index here),

$$
\begin{aligned}
& a_{2}=X_{12} ; \quad a_{1}=2\left(X_{123} p_{1}+X_{213} p_{2}\right) ; \\
& a_{0}=X_{23} p_{1}^{2}+X_{13} p_{2}^{2}+2 X_{132} p_{1} p_{2}-\operatorname{det} \chi .
\end{aligned}
$$

The matrix $\mathbf{W}$ is resolved as

$$
\mathbf{W}=\mathbf{A} q^{2}+\mathbf{B} q+\mathbf{C}+\frac{1}{q^{2}+a q+b}\left(\mathbf{D}^{\prime} q+\mathbf{E}^{\prime}\right),
$$

where, $a=a_{1} / a_{2}, b=a_{0} / a_{2}$. The coefficient matrices are expressed in Appendix A. The condition (8) for nontrivial solution is solved (Sharma, 2007) into eighth-degree polynomial equation

$$
\sum_{j=0}^{8} C_{j} q^{j}=0 .
$$

The coefficients $C_{j}$ are expressed in Appendix B. For the presence of symmetry (monoclinic or higher) in the anisotropy, the coefficients $C_{j},(j=1,3,5,7)$, vanish and Eq. (12) becomes a quartic in $q^{2}$ and hence may be solved algebraically. In any case, this equation is solved for eight values of $q$, with the given values of $p_{1}, p_{2}$. The Snell's law ensures that $\left(p_{1}, p_{2}\right)$ are same for all waves in the medium. The different waves are identified with different vertical slowness values $(q)$. So, the eight complex values of vertical slowness represent the upward (negative $x_{3}$-direction) and downward (positive $x_{3}$-direction) propagation and decay of four waves.

\section{Reflection and Refraction}

\subsection{Geometry of the medium (Fig. 1)}

Consider a fluid half-space in contact with anisotropic poroelastic half-space along a plane interface. In a rectangular co-ordinate system $\left(x_{1}, x_{2}, x_{3}\right)$, the plane $x_{3}=0$ represents the interface between two half-spaces and $x_{3}$-axis is pointing downward into the porous medium. An acoustic wave travels through the fluid and becomes incident at a point on the interface. With reference to a local spherical co-ordinate system having origin at that point, let $(\theta, \phi)$ describes a general direction in the medium. $\theta$ is the polar angle with $x_{3}$-direction and azimuth $\phi$ is measured from (100) to (010) direction. This incident wave, propagating along $\left(\theta_{0}, \phi_{0}\right)$, results in a reflected wave and four attenuating waves refracted to the poroelastic medium. Snell's law ensures that the vertical plane $\phi=\phi_{\mathrm{o}}$ contains the phase direction of all the reflected and refracted waves.

\subsection{Refracted waves}

If $\hat{\mathbf{n}}=\left(n_{1}, n_{2}, n_{3}\right)$ is the direction of propagation, then for incident $P$ wave $p_{j}=n_{j} / v_{\mathrm{o}},(j=1,2)$ and its vertical slowness is given by $q_{\mathrm{o}}=p_{3}=n_{3} / v_{\mathrm{o}}$, where $v_{\mathrm{o}}$ is

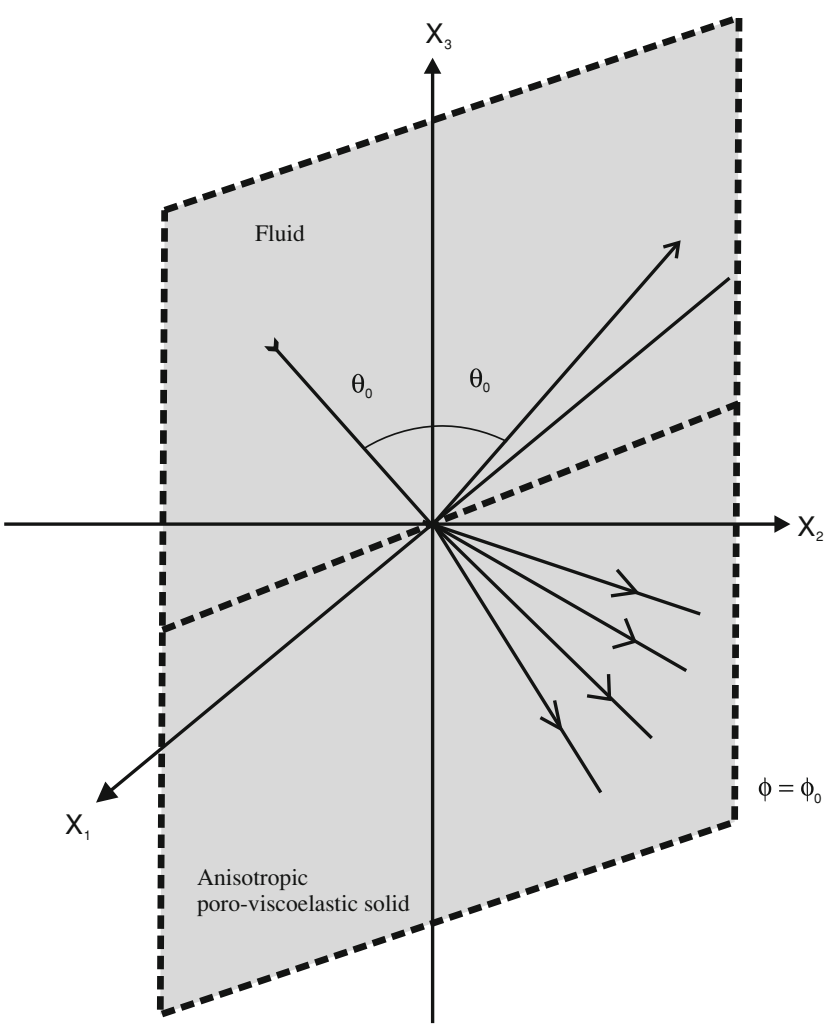

Fig. 1. Geometry of the medium. 
the velocity of sound in fluid half-space. The incident wave is a non-attenuating wave, so $p_{1}, p_{2}$ are real. These values of $p_{1}$ and $p_{2}$ are used to calculate the eight values of $q$ from (11). These values are complex and, hence, the corresponding slowness vectors $\left(p_{1}, p_{2}, q\right)$ represent the propagation and attenuation (along $x_{3}$-direction) of four waves in the medium. Only four among the eight values of $q$ correspond to four waves refracted to the porous medium. Following Carcione (2001), in case of attenuating waves (i.e., complex $q$ ), the values of $q$ for refracted waves are chosen to satisfy the decay (radiation) condition. In the chosen geometry of the problem, positive value of the imaginary part of $q$ ensures the decay condition for a wave in dissipative porous half-space. Thus, the slowness values corresponding to the refracted waves are identified. It is expected that there should be, exactly, four such slowness values $\left(q_{j}, j=1,2,3,4\right)$ for each angle of incidence. The values $j=1,2,3,4$ correspond to $q P_{1}, q s_{1}, q S_{2}, q P_{2}$ waves, respectively. The propagation part $\left(p_{1}, p_{2}, \Re q\right)$ of the slowness vector defines the propagation direction and phase velocity (inverse of magnitude) of the refracted wave. The quality factors $Q$ of attenuation are defined by $Q^{-1}=\Im\left(q^{2}\right) /\left(p_{1}^{2}+p_{2}^{2}+\Re\left(q^{2}\right)\right)$.

\subsection{Displacements and stresses}

The displacement components in the fluid medium are written as

$$
\begin{aligned}
u_{j}^{\mathrm{o}}= & n_{j} \exp \left\{l \omega\left(\frac{1}{v_{\mathrm{o}}} n_{k} x_{k}-t\right)\right\} \\
& +a_{\mathrm{R}} n_{j}^{\prime} \exp \left\{l \omega\left(\frac{1}{v_{\mathrm{o}}} n_{k}^{\prime} x_{k}-t\right)\right\},(j=1,2,3),
\end{aligned}
$$

where $\left(n_{1}, n_{2}, n_{3}\right)=\left(\sin \theta_{\mathrm{o}} \cos \phi_{\mathrm{o}}, \sin \theta_{\mathrm{o}} \sin \phi_{\mathrm{o}}, \cos \theta_{\mathrm{o}}\right)$ and $\left(n_{1}^{\prime}, n_{2}^{\prime}, n_{3}^{\prime}\right)=\left(\sin \theta_{0} \cos \phi_{0}, \sin \theta_{0} \sin \phi_{0},-\cos \theta_{0}\right)$. The normal stress in the fluid is given by

$$
\sigma_{33}=\rho_{\mathrm{o}} v_{\mathrm{o}}^{2} u_{j, j}^{\mathrm{o}}
$$

The displacement components in the anisotropic poroelastic medium are expressed as

$$
\begin{array}{r}
u_{j}=\sum_{m=1}^{4} a(m) S_{j}^{(m)} \exp \left\{l \omega\left(p_{k}^{(m)} x_{k}-t\right)\right\} ; \\
w_{j}=\sum_{m=1}^{4} a(m) F_{j}^{(m)} \exp \left\{l \omega\left(p_{k}^{(m)} x_{k}-t\right)\right\} ; \\
(j=1,2,3),
\end{array}
$$

where, $\left(p_{1}^{(m)}, p_{2}^{(m)}, p_{3}^{(m)}\right)=\left(n_{1} / v_{0}, n_{2} / v_{0}, q_{m}\right)$ is the slowness vector for refracted wave ' $m$ '. The $a(m)$ are relative excitation factors. The stresses in the porous aggregate and pore-fluid pressure are expressed as

$$
\tau_{i j}=c_{i j k l} u_{k, l}+m_{i j} w_{k, k}, \quad-p_{\mathrm{f}}=m_{i j} u_{i, j}+R w_{k, k} .
$$

\subsection{Boundary conditions}

Following Deresiewicz and Skalak (1963), the boundary conditions at the interface between a fluid medium and a poroelastic medium are the continuity of stresses. Continuity of normal components of velocity is also considered.
In porous medium, the flow of viscous pore-fluid in pores makes the wave propagation dispersive and is responsible for the dissipation of energy during propagation. The flow condition at a saturated porous solid interface might play a key role in the reflection and transmission of waves (Yang, 1999). In this paper, following are the five appropriate boundary conditions, which are required to be satisfied at the plane $x_{3}=0$.

$$
\begin{aligned}
& \text { i) } \tau_{31}=0, \quad \text { ii) } \tau_{32}=0, \text { iii) } \tau_{33}=\sigma_{33}, \\
& \text { iv) } \xi\left(-p_{\mathrm{f}}-\tau_{33}\right)=(1-\xi) Z \dot{w}_{3}, \\
& \text { v) } \dot{u}_{3}+\dot{w}_{3}=\dot{u}_{3}^{\mathrm{o}},
\end{aligned}
$$

where, $Z$ (a constant) may be termed as a surface flow impedance for the pore-fluid (Denneman et al., 2002). The value $Z=0$ corresponds to fully open pores and the limit $Z \rightarrow \infty$ corresponds to sealed pores.

Another parameter $\xi$ is used to represent the partial opening of surface pores of the porous bed. Then, $Z$ will be assumed a non-zero, finite constant value for surface flow impedance. The extreme values of $\xi$, i.e., 0 and 1 , denote sealed pores and open pores, respectively. The intermediate values of $\xi$ may represent some restricted or partial opening of pores. The use of $\xi$, as above, has a special significance. This implies that even a small or restricted opening of the pores at the interface may cause a large drop in the normal stress of pore-fluid.

Satisfying the above boundary conditions with the displacements defined in Section 3.3, yields a system of five linear inhomogeneous equations in $a(1), a(2), a(3), a(4)$ and $a_{\mathrm{R}}$. These equations are given by

$$
\begin{aligned}
& \sum_{m=1}^{4} J_{i}^{(m)} a(m)-\rho_{\mathrm{o}} v_{\mathrm{o}} \delta_{i 3} a_{\mathrm{R}}=\rho_{\mathrm{o}} v_{\mathrm{o}} \delta_{i 3}, \quad(i=1,2,3) ; \\
& \sum_{m=1}^{4}\left[(1-\xi) Z F_{3}^{(m)}-\xi J_{4}^{(m)}\right] a(m)=0, \\
& \sum_{m=1}^{4}\left(S_{3}^{(m)}+F_{3}^{(m)}\right) a(m)+n_{3} a_{\mathrm{R}}=n_{3},
\end{aligned}
$$

where,

$$
\begin{aligned}
& J_{i}^{(m)}=c_{3 i k l} p_{l}^{(m)} S_{k}^{(m)}+m_{3 i} p_{l}^{(m)} F_{l}^{(m)}, \\
& J_{4}^{(m)}=J_{3}^{(m)}-m_{k l} S_{k}^{(m)} p_{l}^{(m)}-R F_{k}^{(m)} p_{k}^{(m)} .
\end{aligned}
$$

The above system of linear equations is solved numerically using the Gauss elimination method. The complex solution of this system explains the amplitudes and phase shifts of the waves scattered at the interface.

\subsection{Energy ratios}

Distribution of energy between different reflected and refracted waves is considered across a surface element of unit area at the plane $x_{3}=0$. The scalar product of surface traction and particle velocity per unit area, denoted by $P^{*}$, represents the rate at which the energy is communicated per unit area of the surface. The time average of $P^{*}$ over a period, denoted by $\left\langle P^{*}\right\rangle$, represents the average energy transmission per unit surface area per unit time. With $x_{3}$ axis as the outer normal to the surface of fluid medium, the 
average energy fluxes of incident and reflected waves are $\left\langle P_{\mathrm{I}}^{*}\right\rangle=-0.5 \omega^{2} \rho_{\mathrm{o}} v_{\mathrm{o}} n_{3}$ and $\left\langle P_{\mathrm{R}}^{*}\right\rangle=-0.5 \omega^{2}\left|a_{\mathrm{R}}\right|^{2} \rho_{\mathrm{o}} v_{\mathrm{o}} n_{3}^{\prime}$, respectively. The ratio $\left\langle P_{\mathrm{R}}^{*}\right\rangle /\left\langle P_{\mathrm{I}}^{*}\right\rangle$ defines the reflection coefficient of the reflected wave as $E_{\mathrm{R}}=\left|a_{\mathrm{R}}\right|^{2}$. The argument of complex number $a_{\mathrm{R}}$ defines the phase shift of the reflected wave.

The waves refracted across the interface propagate as inhomogeneous waves in a dissipative porous medium and hence involve the existence of interaction energy (Borcherdt, 1977) amongst them. To ensure energy conservation, Ainslie and Burns (1995) have also explained some derivations involving interference energy or complex energy ratios. In the present problem, an energy matrix $E_{j k}=\left\langle P_{j k}^{*}\right\rangle /\left\langle P_{\mathrm{I}}^{*}\right\rangle ;(j, k=1,2,3,4)$, is defined to calculate the interaction energy among the four waves in poroelastic medium. The average energy fluxes in the porous medium are defined as

$$
\begin{aligned}
\left\langle P_{j k}^{*}\right\rangle= & -0.5 \omega^{2} \Re\left\{\sum_{i=1}^{3} J_{i}^{(j)} a(j) \bar{S}_{i}^{(k)} \bar{a}(k)\right. \\
& \left.+J_{4}^{(j)} a(j) \bar{F}_{3}^{(k)} \bar{a}(k)\right\} .
\end{aligned}
$$

The sum of all the non-diagonal entries of the energy matrix $\left\{E_{j k}\right\}$ gives the share of interaction energy for the refracted waves. The diagonal entries of this matrix represent the energy ratios of the transmitted waves in the porous medium. The conservation of energy is obtained through the relation $\sum_{j=1}^{4} \sum_{k=1}^{4} E_{j k}+E_{\mathrm{R}}=1$.

\section{Numerical Examples}

The analytical expressions derived in previous sections define a general mathematical model for reflectionrefraction phenomenon at an interface between inviscid fluid half-space resting over anisotropic porous solid saturated with a viscous fluid. The mathematical model may be used to study the effects of elastic/hydraulic anisotropy, pore-fluid-viscosity, frame anelasticity, frequency, porosity, etc., on the propagation characteristics (phase velocity, group velocity, ray direction, energy flux, spatial dispersion, slowness, attenuation, inhomogeneity, fluid/solid polarisations) of the waves scattered into two continuing media. The present numerical work is, however, restricted to analyse the energy partition of acoustic waves incident through the inviscid fluid among the resulting reflected and refracted waves. The ocean bed is the most suitable anisotropic porous medium to be used in this numerical example. North-Sea Sandstone (Rasolofosaon and Zinszner, 2002) is assumed a porous aggregate of density $2216 \mathrm{~kg} / \mathrm{m}^{3}$ that contains $15.7 \%$ pore space filled with a fluid of density $980 \mathrm{~kg} / \mathrm{m}^{3}$ and $R=5 \mathrm{GPa}$. The elastic matrix (in GPa) for North-Sea Sandstone is given by $c_{11}=17.77 c_{12}=3.78 \quad c_{13}=3.76 c_{14}=0.24 Z_{1} c_{15}=$ $-0.28 Z_{1} \quad c_{16}=0.03 Z_{2} ; c_{22}=19.45 \quad c_{23}=4.13 \quad c_{24}=$ $-0.41 Z_{1} \quad c_{25}=0.07 Z_{1} \quad c_{26}=1.13 Z_{2} ; c_{33}=21.79 c_{34}=$ $-0.12 Z_{1} \quad c_{35}=-0.01 Z_{1} \quad c_{36}=0.38 Z_{2} ; c_{44}=8.30 c_{45}=$ $0.66 Z_{2} \quad c_{46}=0.06 Z_{1} \quad c_{55}=7.62 \quad c_{56}=0.52 Z_{1} \quad b_{66}=$ 7.77, where, $c_{i j}$ denote the two-suffix notations (Crampin, 1989) for elastic tensor $c_{i j k l}$ of porous aggregate. The symmetric tensor of anisotropic permeability is given by, $\chi_{\mathrm{a}}=\left\{0.81,0.28 Z_{2},-0.04 Z_{1} ; 0.28 Z_{2}, 0.52,-0.1 Z_{1}\right.$; $\left.-0.04 Z_{1},-0.1 Z_{1}, 0.73\right\}$. Anisotropic elastic coupling is assumed through the symmetric matrix $\mathbf{M}=R\left\{1,-0.1 Z_{2}\right.$, $\left.0.2 Z_{1} ;-0.1 Z_{2}, 1.1,0.15 Z_{1} ; 0.2 Z_{1}, 0.15 Z_{1}, 0.9\right\}$. These anisotropic tensors with value of $Z_{1}=Z_{2}=1$ define the triclinic system of anisotropy. The monoclinic symmetry is represented by $Z_{1}=0, Z_{2}=1$ and the values $Z_{1}=Z_{2}=0$ represent the orthotropic symmetry. For overlying water half-space density $\rho_{\mathrm{o}}=1000 \mathrm{~kg} / \mathrm{m}^{3}$ and velocity $v_{\mathrm{o}}=1463 \mathrm{~m} / \mathrm{s}$.

The above numerical values are used to calculate the energy partition according to the relations derived in the previous section. Various numerical models are used to compute and analyse the effects of geophysical properties (anisotropy, anelasticity, pore-characteristics) of porous medium and frequency of wave propagation on the energy shares of waves reflecting back in liquid half-space as well as of waves refracting to the ocean bed. The conservation of energy is observed and verified for all the models. The detailed variations of reflected energy with incident angle (from 0 to $90^{\circ}$ ) are discussed as follows.

\subsection{Reference media}

For incidence of an acoustic wave through liquid halfspace at its interface with isotropic poroelastic solid halfspace, three waves are refracted alongwith one reflected wave. Energy shares of these waves are calculated for different kinds of opening of surface pores and are exhibited in Fig. 2 for direction of incidence $\left(\theta_{\mathrm{I}}\right)$ varying from 0 to $90^{\circ}$. In this figure, reflection and refraction coefficients are shown for the situations, fully open pores $(\xi=1)$ sealed pores $(\xi=0)$ and partially open pores $(0<\xi<1)$. Except for $P_{\mathrm{s}}$ wave, the energy shares of all the waves decrease with the opening of pores at the ocean bottom. Energy partition in this figure will serve as a platform to analyse the effects of some attributes (like, anisotropy, anelasticity, tortuosity of pores, viscosity of pore-fluid, etc.) of realistic model of porous bed considered in the study. Similarly, energy partition at the interface between fluid and anisotropic elastic solid is shown in Fig. 3, for different propagation planes. The plots in this figure are used to identify the effects of porosity on reflected/refracted waves in composite anisotropic medium. Anisotropy considered for the computation, except for Figs. 1 and 5, is triclinic anisotropy.

\subsection{Porosity}

In comparison to Fig. 3, Fig. 4 has two extra plots. One shows the interaction energy $\left(E_{\mathrm{I}}\right)$ among the refracted waves, which is zero in this non-dissipative medium. The other plot shows the variation of energy share of refracted $q P_{2}$ wave, which is the diagnostic feature of wave propagation in a saturated porous solid. So the presence of saturated pores results in an additional wave. In Fig. 3, the complete reflection is observed for incidence beyond the critical angles of three refracted waves (i.e., $\theta_{\mathrm{I}}>55^{\circ}$ in $\phi=0$ plane). However, in the presence of saturated pores (Fig. 4), the similar complete reflection is observed for incidence beyond $\theta_{\mathrm{I}}>70^{\circ}$, because critical angle for refracted $q P_{2}$ wave is observed around $70^{\circ}$ in $\phi=0$ plane. So, for incidence between $55^{\circ}$ to $70^{\circ}$, the refracted $q S_{2}$ wave is showing its strong presence as well as variations. Variations of energy ratios with the change of vertical planes (i.e., $\phi)$ rep- 

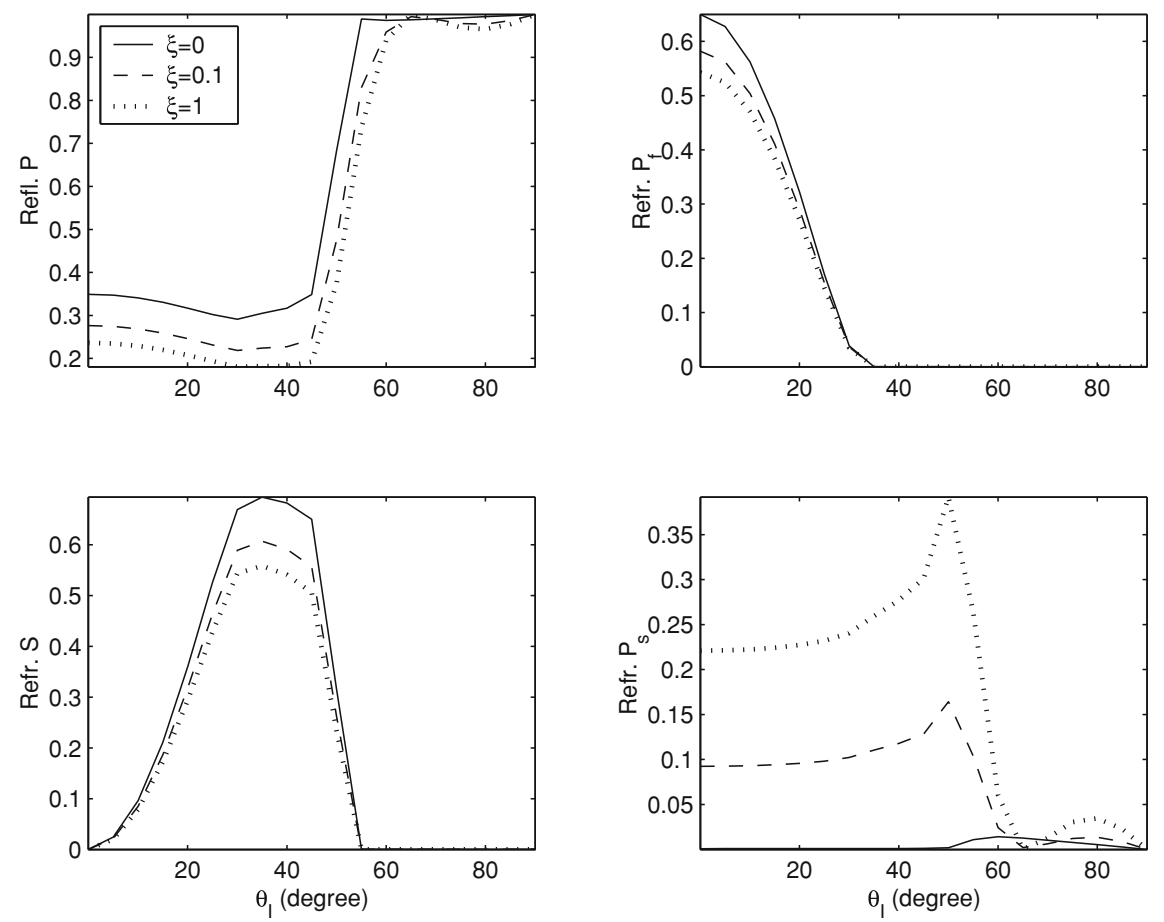

Fig. 2. Energy shares of reflected $(P)$ wave and refracted $\left(P_{\mathrm{f}}, P_{\mathrm{s}}, S\right)$ waves at the boundary of isotropic porous solid saturated with inviscid fluid; variation with surface pore opening: $\xi=0,0.1,1 ; \alpha_{\infty}=6.2, Z=1 \mathrm{~N} \mathrm{~s} / \mathrm{m}, \epsilon=0$.
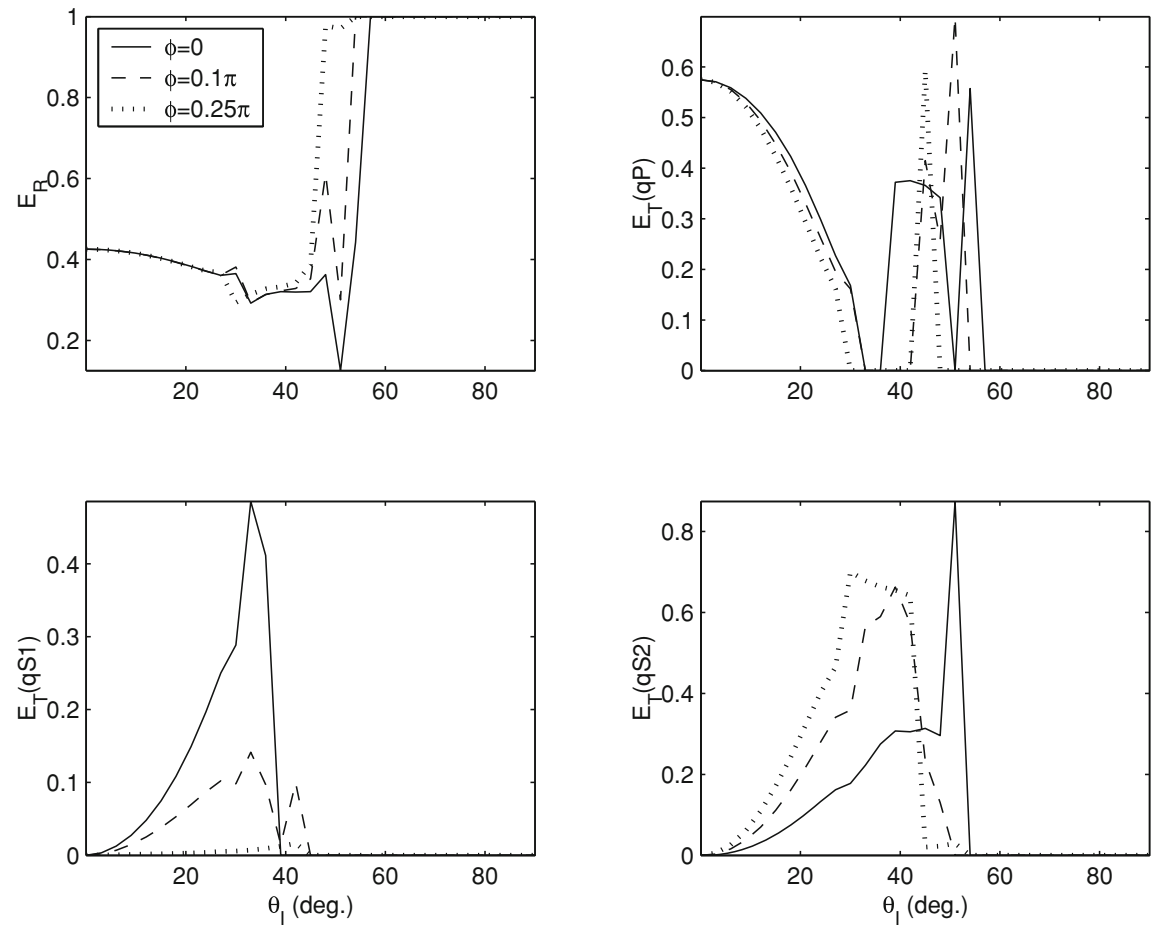

Fig. 3. Energy shares of reflected $(P)$ wave $\left(E_{\mathrm{R}}\right)$ and refracted $\left(q P, q S_{1}, q S_{2}\right)$ waves $\left(E_{\mathrm{T}}\right)$ at the boundary of (triclinic) anisotropic elastic medium; variation with propagation plane: $\phi=0,0.1 \pi, 0.25 \pi ; \epsilon=0$.

resent the effect of azimuthal anisotropy of porous medium on the reflected and refracted waves.

\subsection{Anisotropy}

Effect of presence of anisotropy in poroelastic solid halfspace is the presence of two split-shear $\left(q S_{1}\right.$ and $\left.q S_{2}\right)$ waves as shown in Fig. 5. Its effect on energy partition, among reflected and refracted waves, is obtained by comparing the curves in Fig. 5 with the dotted curves (fully open surface pores) in the corresponding plots of Fig. 2. Major difference comes in the form of two critical regions for refracted $q P_{1}$ wave. For incidence directions in these regions, there is no refracted $q P_{1}$ wave whereas, in case of isotropic porous solid, there exists only one such region (i.e., beyond the critical angle of refracted $P$ wave). Other major effect is 

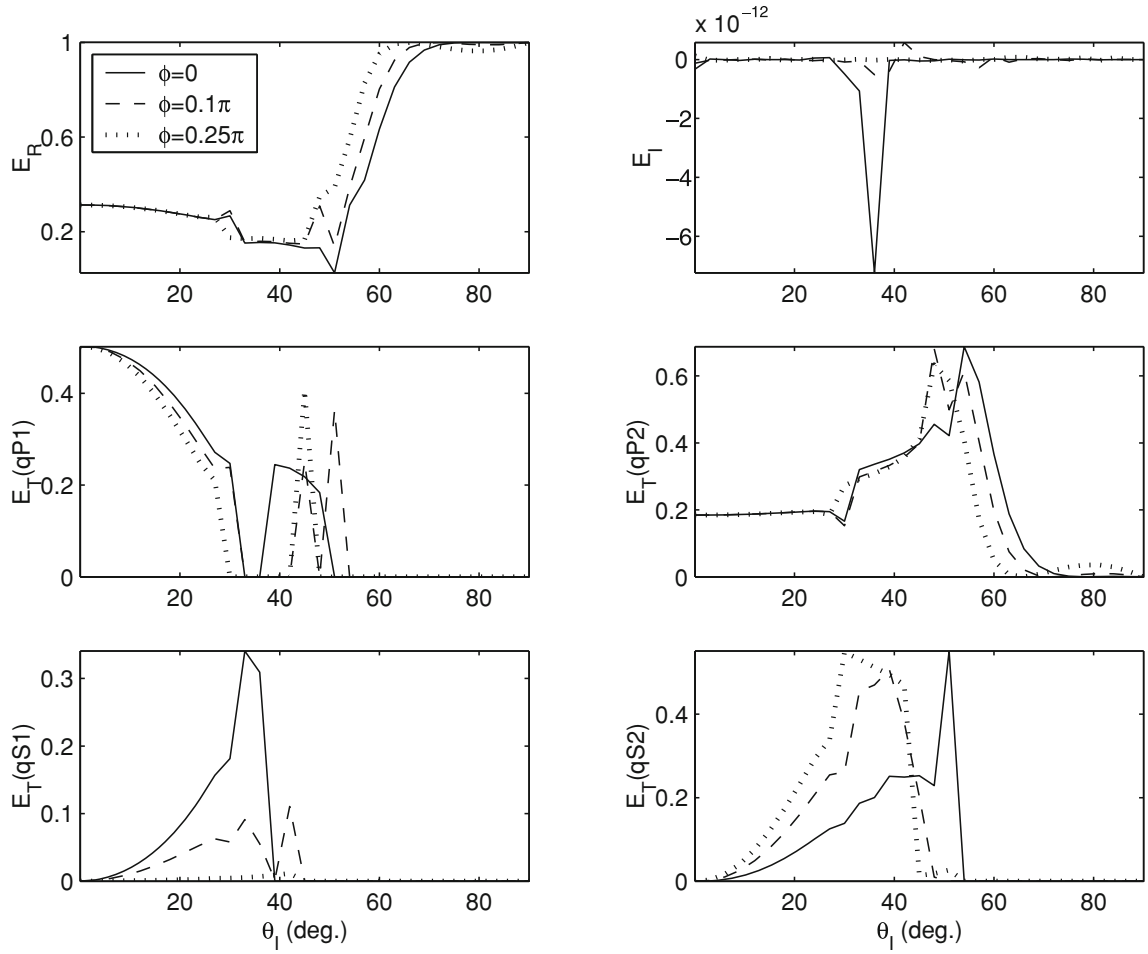

Fig. 4. Energy shares of reflected $(P)$ wave $\left(E_{\mathrm{R}}\right)$, refracted $\left(q P_{1}, q P_{2}, q S_{1}, q S_{2}\right)$ waves $\left(E_{\mathrm{T}}\right)$ and interaction energy $\left(E_{\mathrm{I}}\right)$ at the boundary of $($ triclinic) anisotropic porous solid saturated with inviscid fluid; variation with propagation plane: $\phi=0,0.1 \pi, 0.25 \pi ; \xi=1, \alpha_{\infty}=6.2, Z=1 \mathrm{~N} \mathrm{~s} / \mathrm{m}$, $\epsilon=0$.
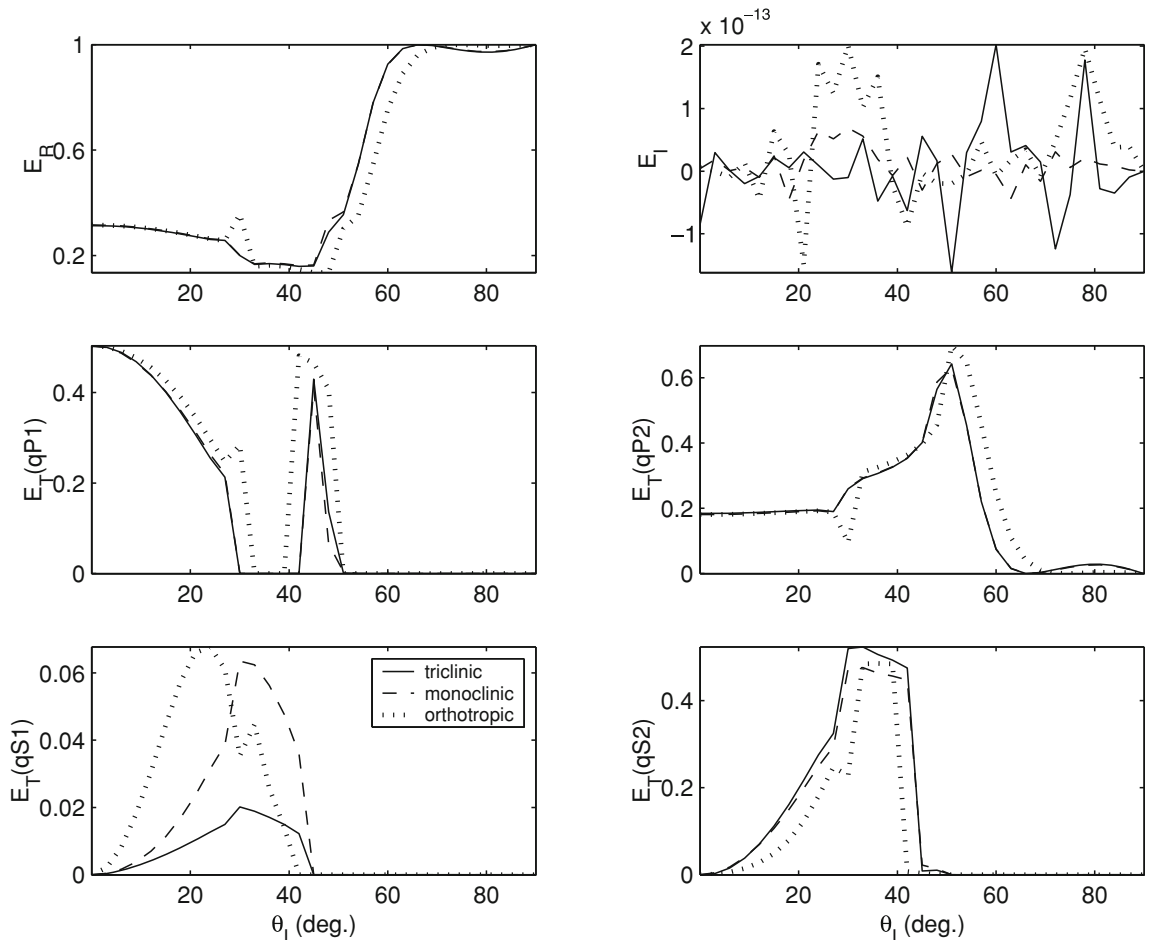

Fig. 5. Same as Fig. 4, but variation with anisotropic symmetries of porous solid: triclinic, monoclinic, orthotropic; $\phi=0.2 \pi, \xi=1, \alpha=6.2, Z=$ $1 \mathrm{~N} \mathrm{~s} / \mathrm{m}, \epsilon=0$.

the strength gained by refracted $q P_{2}$ wave for incidence between $40^{\circ}$ to $60^{\circ}$ as compared to that of $P_{\mathrm{s}}$ wave in Fig. 2 . Due to this effect the incidence direction for complete reflection of $P$ wave appears beyond $60^{\circ}$. The energy of refracted lone $S$ wave in Fig. 2 appears to be distributed be- tween two split-shear waves in Fig. 5. The presence of monoclinic symmetry in the anisotropy of porous medium have a quite significant effect only on split-shear waves. However, the presence of orthotropic symmetry do affect the energy shares of reflected and all the refracted waves. It 

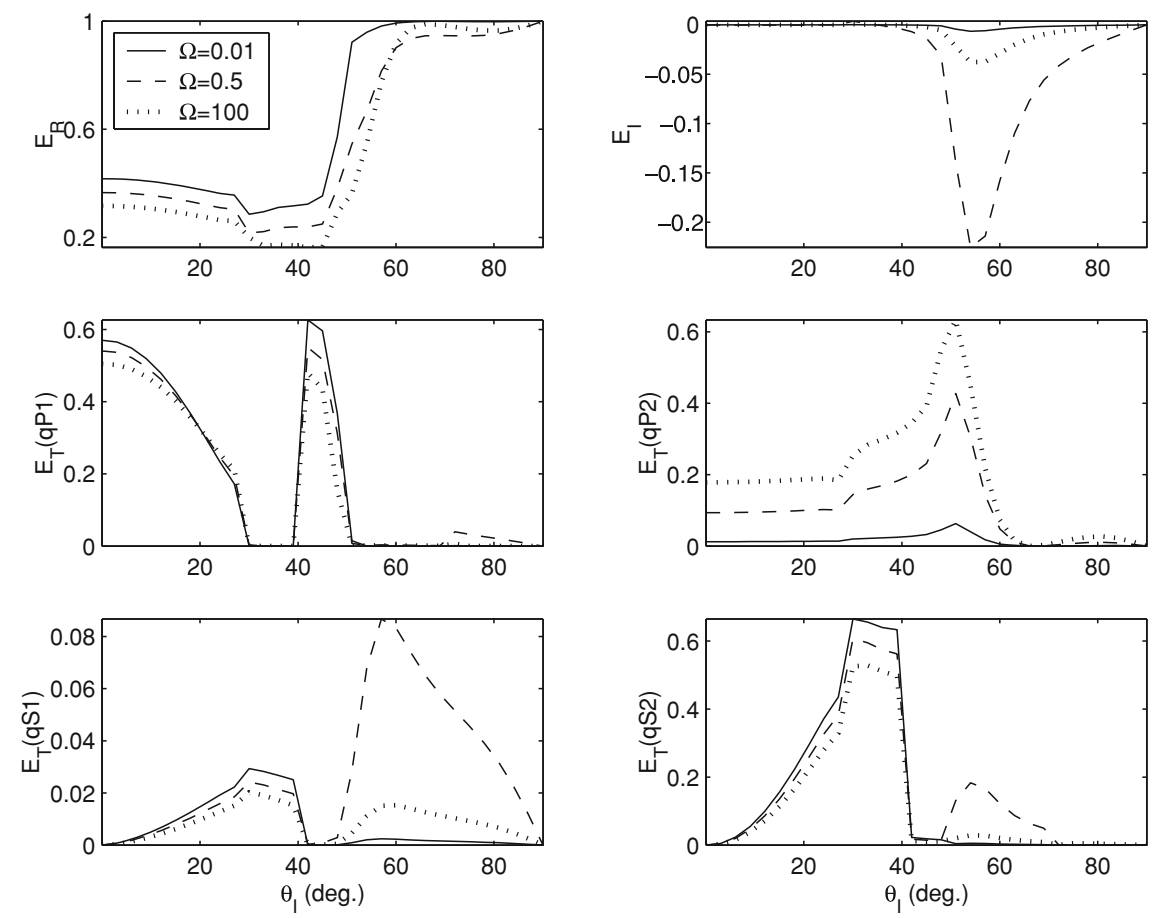

Fig. 6. Same as Fig. 4. but anisotropic porous solid is saturated with viscous fluid; variation with frequency: $\Omega=0.01$, 0.5 , 100; $\phi=0.2 \pi, \xi=1, \alpha_{\infty}=6.2, Z=1 \mathrm{~N} \mathrm{~s} / \mathrm{m}, \epsilon=0$.
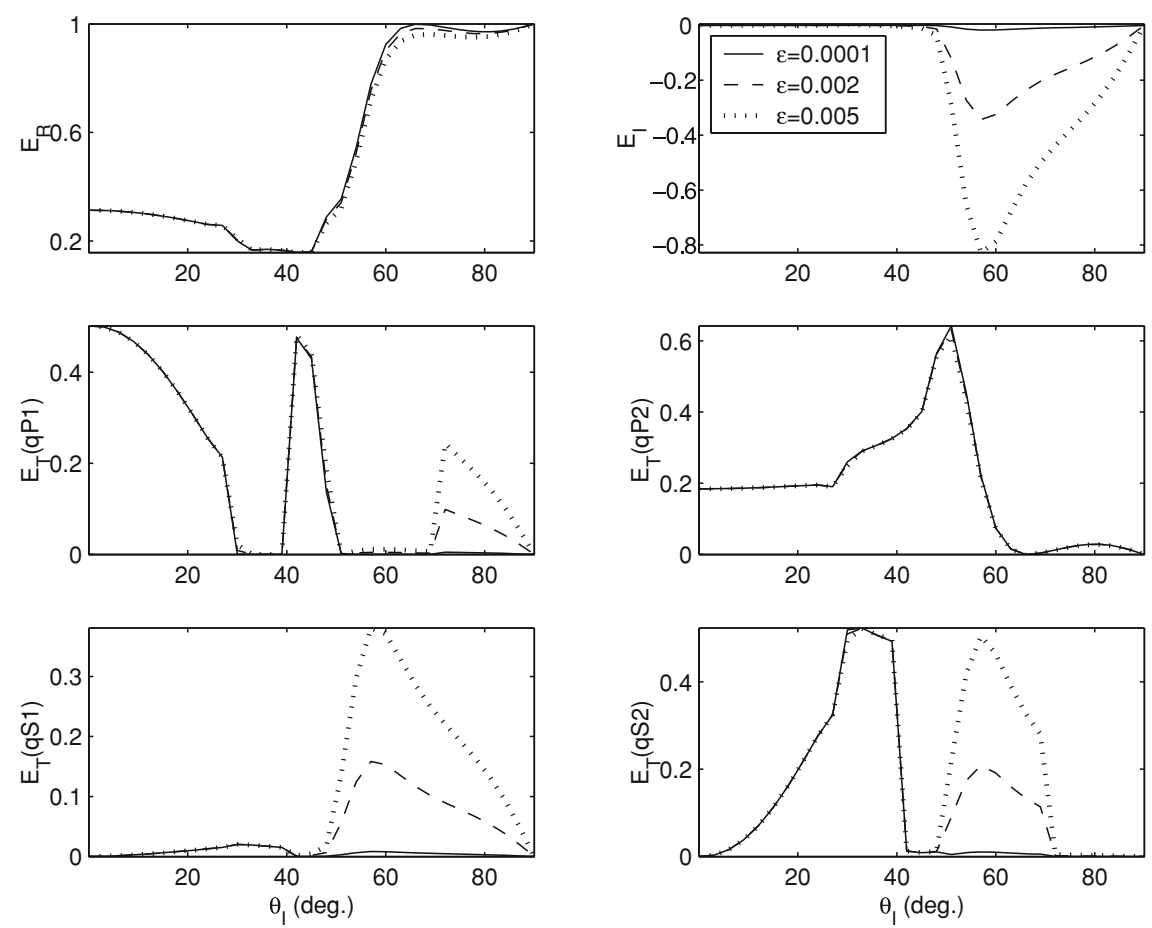

Fig. 7. Same as Fig. 4, but for anelastic porous solid frame: $\epsilon=0.0001,0.002,0.005 ; \phi=0.2 \pi, \xi=1, \alpha_{\infty}=6.2, Z=1 \mathrm{~N}$ s $/ \mathrm{m}$.

strongly affects the critical angles of refracted waves.

\subsection{Pore-fluid viscosity}

Plots in Fig. 6, when compared with the solid curves of the corresponding plots in Fig. 5, provide the effects of pore-fluid viscosity on energy partition. Major effect is the presence of interaction energy $\left(E_{\mathrm{I}}\right)$. Negative sign of this energy implies that aggregate energy of interaction among the refracted waves in porous media is towards the interface. This implies that some of the waves moving away from the interface (e.g., $q P_{1}$ and $q S_{1}$ ) may be getting stronger with the presence of viscosity in the pore fluid. Another aspect of plots in Fig. 6 is to explore the role the wave-frequency plays in changing the strengths of reflected/refracted signals. It is observed that reflected wave is stronger in low-frequency regime and weakens with the increase of frequency. This loss of reflected wave in high- 

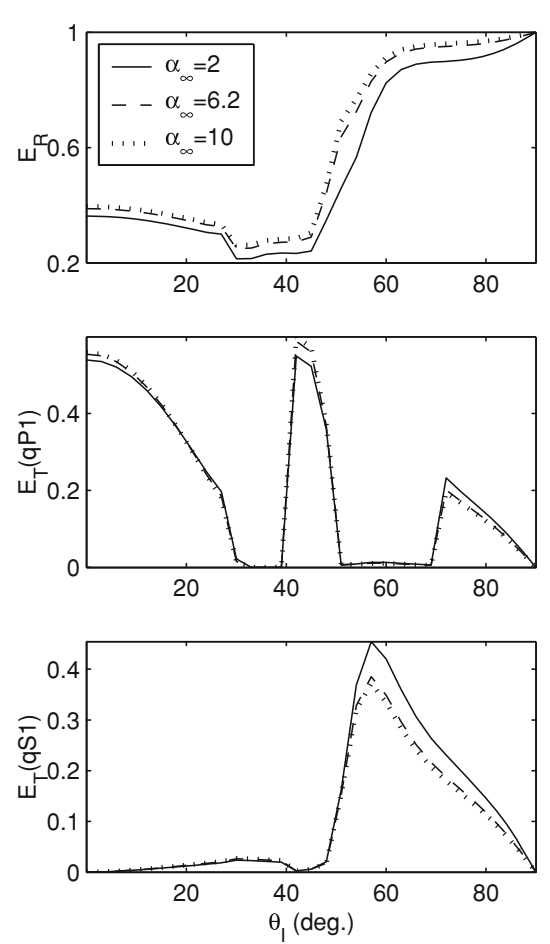
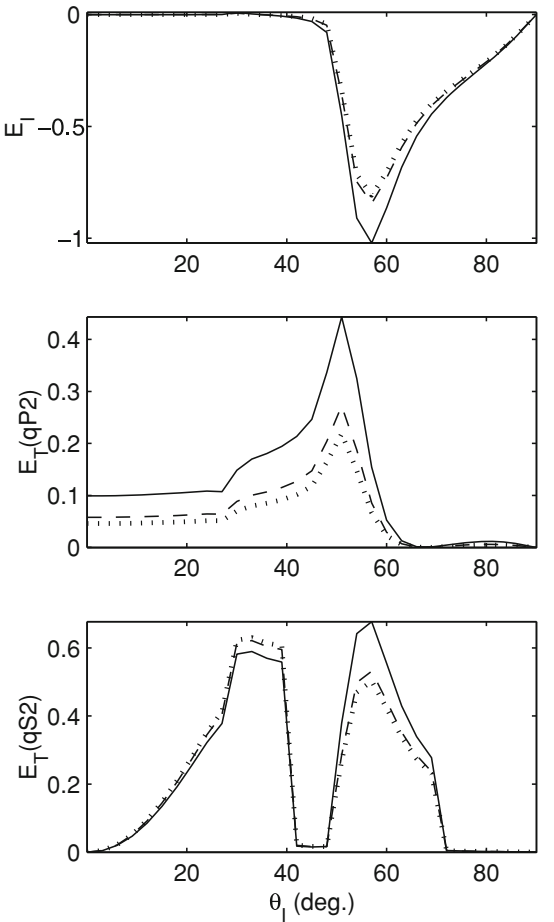

Fig. 8. Same as Fig. 6, but porous solid frame is anelastic with $\epsilon=0.004$; variation with pore tortuosity: $\alpha_{\infty}=2$, 6.2, 10; $\Omega=0.2, \phi=0.2 \pi, \xi=1, Z=1 \mathrm{~N} \mathrm{~s} / \mathrm{m}$.

frequency regime is appeared to be the gain of refracted $q P_{2}$ wave. It is a known fact that the second dilatation wave of Biot's theory is a diffusion type wave in low-frequency regime and it becomes a propagating wave in the high frequency regime. The refracted $q P_{1}$ and $q S_{2}$ waves may weaken a little with the increase of frequency. The interaction energy is more prominent when the waves propagate at intermediate frequencies and this energy change is adjusted in the increase of the energy shares of $q S_{1}$ and $q S_{2}$ waves.

\subsection{Frame anelasticity}

Compare the plots in Fig. 7 to the solid curves in the corresponding plots in Fig. 5 to explore the effects of porousframe anelasticity on the energy partition at the interface. The reflected wave and refracted $q P_{2}$ wave seem to be nearly unaffected. Other refracted waves are affected significantly but for incidence beyond their critical angles in Fig. 5. On the other hand, Fig. 7 explains the effect of increase in anelastic coefficient $(\epsilon)$ on the energy shares of refracted waves. Increase in $\epsilon$ increases the energy shares of refracted $q P_{1}, q S_{1}, q S_{2}$ waves but only after incidence beyond their critical angles. This increase is balanced by the negative increase in interaction energy and the conservation of energy is ensured at the interface. Absence of critical angle for refracted $q P_{2}$ wave is responsible for the immunity of this wave to anelasticity of the frame.

\subsection{Tortuosity}

Figure 8 presents the effect of tortuosity of pores on the energy shares of reflected and refracted waves at the interface. It is observed that the reflected waves are getting stronger with the increase in tortuosity $\left(\alpha_{\infty}\right)$. The effect is also observed on all the refracted waves but the most affected is $q P_{2}$ wave, which loses much of its energy with the increase of tortuosity in pores. Interaction energy decreases with the increase of $\alpha_{\infty}$ but for incidence between $45^{\circ}$ and $75^{\circ}$. Other aspect of this figure is to explore the effect of anelasticity of porous frame in the presence of pore-fluid dissipation. This is obtained by comparing the dashed curves in this figure to the dashed curves in the corresponding plots in Fig. 6. It is observed that the effect of anelasticity (mainly on $q P_{1}, q S_{1}, q S_{2}$ waves) is nearly a superposition on the effect pore-fluid dissipation (mainly on $q P_{2}$ wave).

\subsection{Surface flow impedance}

There may be some other important aspects, which can affect the energy partition at the plane surface of ocean bottom. These aspects represent the behaviour of surface pores of the saturated porous bed in contact with fluid and are significant more at high frequencies. One of them is the presence of impedance $(Z)$, which accounts for the obstruction in fluid discharge out of porous medium due to the partial opening of surface pores. Figure 9 exhibits the variations in energy shares of reflected and refracted waves with the change in the value of impedance. The increase of $Z$ strengthens the reflected $P$ wave but weakens the refracted $q P_{2}$ wave. For much larger value of $Z$, the $q P_{2}$ wave may not remain noticeable in porous bed, i.e., saturated porous bed with high impedance (nearly sealed surface pore situation) to surface flow may behave like an elastic solid. Effect on other waves may be significant but only for few incident directions. The conservation of energy is possible only for extreme values of $Z=0$ and $Z \rightarrow \infty$. For non-zero finite values of $Z$, a part $\left(E_{\mathrm{D}}\right)$ of incident energy is dissipated due to this surface flow impedance and this part increases with the deviation in value of $Z$ from any of the extremes. 

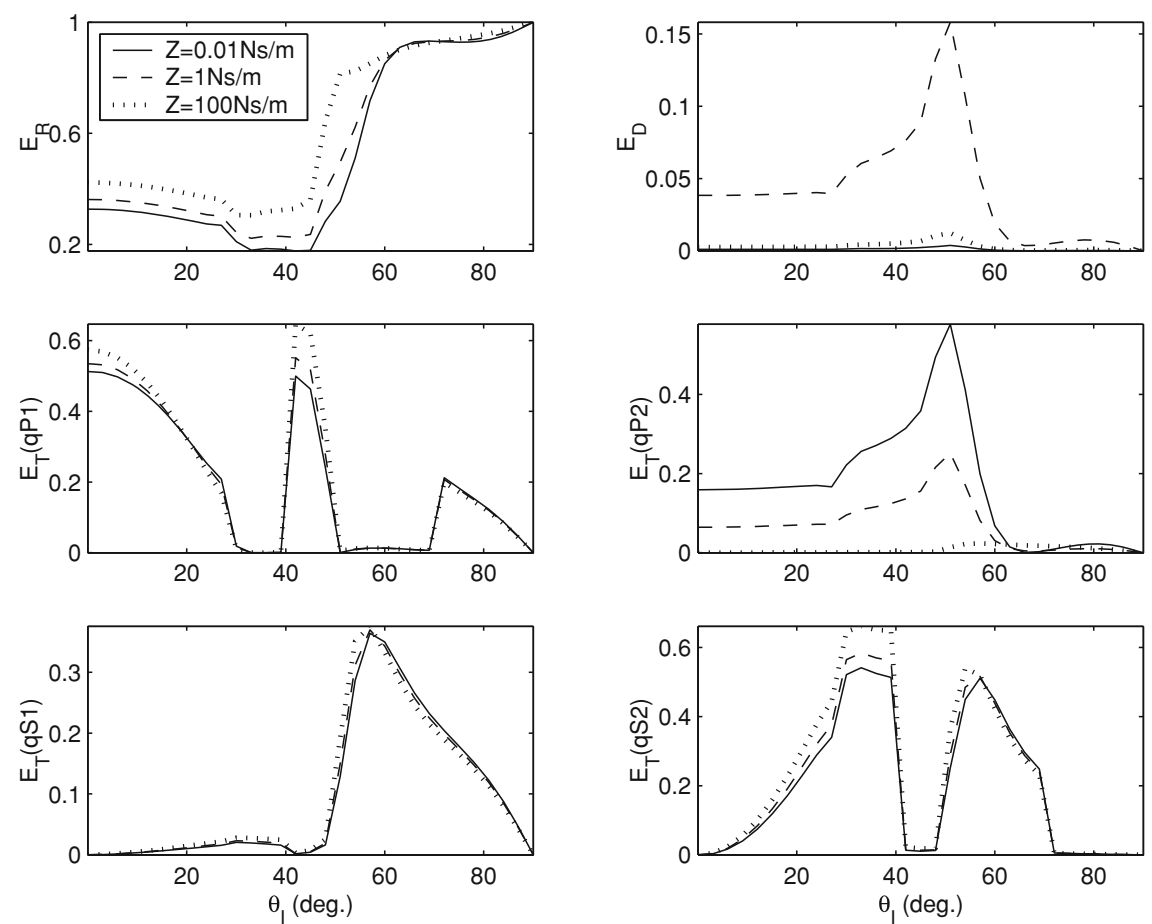

Fig. 9. Energy shares of reflected $(P)$ wave $\left(E_{\mathrm{R}}\right)$, refracted $\left(q P_{1}, q P_{2}, q S_{1}, q S_{2}\right)$ waves $\left(E_{\mathrm{T}}\right)$ and dissipated energy $\left(E_{\mathrm{D}}\right)$ at the boundary of (triclinic) anisotropic porous anelastic solid saturated by viscous fluid; partial pore opening $\xi=0.1$; variation with surface flow impedance: $Z=0.01,1,100 \mathrm{~N} \mathrm{~s} / \mathrm{m} ; \epsilon=0.004, \alpha_{\infty}=6.2, \Omega=5, \phi=0.2 \pi$.
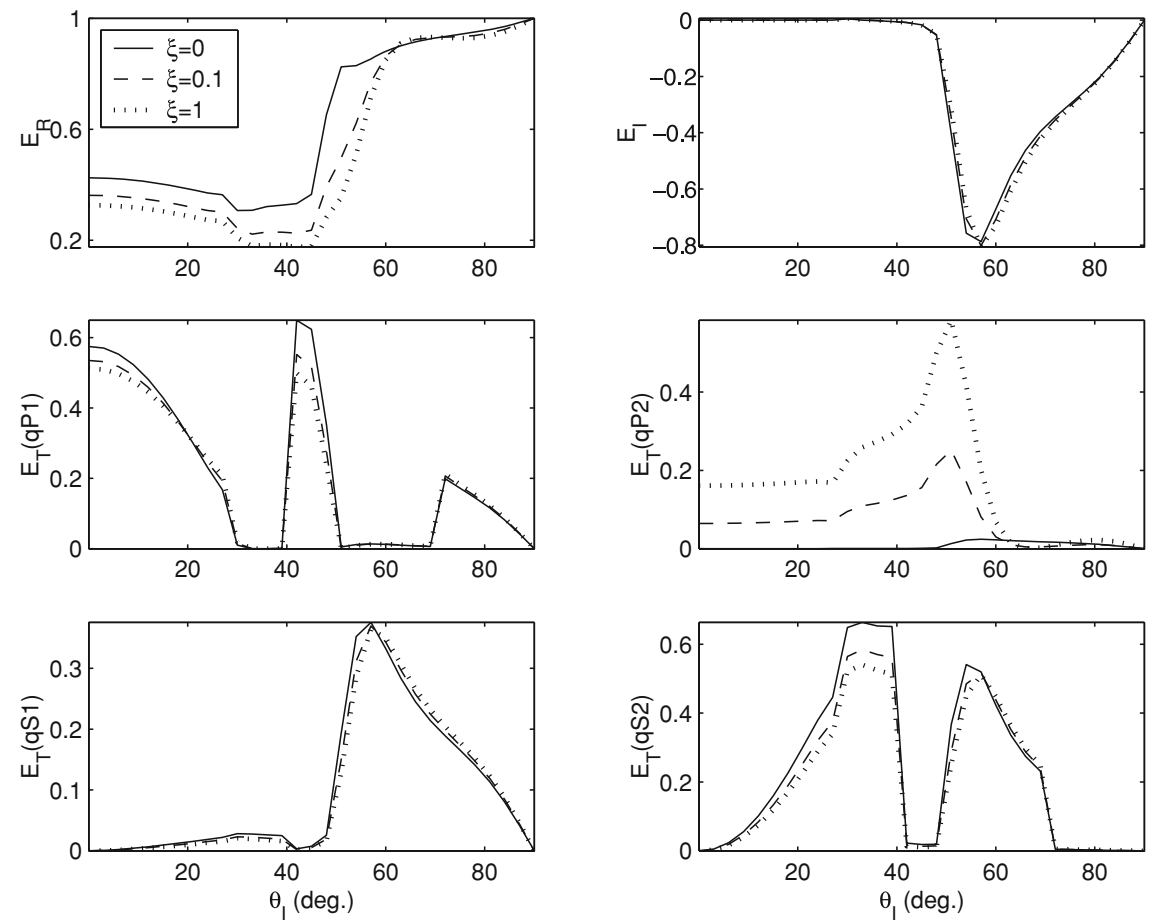

Fig. 10. Same as Fig. 8, but variation with surface pore opening: $\xi=0,0.1,1 ; Z=1 \mathrm{~N} \mathrm{~s} / \mathrm{m}, \alpha_{\infty}=6.2, \Omega=5, \phi=0.2 \pi$.

\subsection{Opening of surface-pores}

Related to surface-flow impedance, another aspect is opening of surface pores, which becomes significant when $Z$ is deviated from its extreme values. Figure 10 exhibits the role of pore opening parameter $\xi$ on energy partition for $Z=1 \mathrm{~N} \mathrm{~s} / \mathrm{m}$. The extreme values of $\xi$, i.e., 0 and 1 , represent closed and fully opened surface pores, respec- tively. It is observed that the reflected wave weakens with the opening of the surface pores. Major effect of pore opening is manifold increase in the energy share of $q P_{2}$ wave at the cost of other refracted waves and reflected $P$ wave. Presence or change in discharge through surface pores may not have much effect on the interaction between refracted waves. 


\section{Concluding Remarks}

This study considers a general and realistic model of ocean bed to analyse the reflection and refraction phenomenon at the ocean bottom. The mathematical model relates the acoustic/seismic response of poroelastic materials to their physical/mechanical properties. In particular, such a composite physical model facilitates the parametric studies of the influence of various measurable physical properties of the medium (i.e., porosity, permeability, pore-fluid viscosity, frame inelasticity, fibre-reinforcements, microcracks, elastic/hydraulic anisotropy, pore-configuration, etc.) on the waves propagating in it. Energy losses, both in the skeletal frame of the sediment and viscous losses in the pore liquid as it moves relative to the frame, have been taken into account by considering the two-component nature of porous materials. The general anisotropy provides complete liberty in choosing the directional dependence of elastic as well as hydraulic properties (permeability) of the medium. Surface flow impedance incorporated in the boundary condition provides an option for resisting the fluid discharge out of porous aggregate. Partial opening of surface pores results in the dissipation of a part of incident energy, to be used in obstructing the free flow of pore fluid.

Numerical examples consider some particular models for the computation of energy partition among reflected and refracted waves. Energy conservation is verified when the surface pores are fully closed or open. Energy dissipation is observed for partial opening of pores at the surface of pores solid. From the discussion of numerical results in previous section, following few points may be noted.

1) $q P_{2}$ wave is the diagnostic feature of the wave propagation in a saturated porous medium. Fortunately, this diagnostic wave refracted to porous bed is very sensitive to the presence of dissipation in pore-fluid.

2) In the presence of dissipation in porous ocean bed due to pore-fluid viscosity, the reflected and refracted waves are dispersive. Reflected $P$ wave is stronger at low frequency, whereas refracted $q P_{2}$ wave gains strength at high frequency. For post-critical incidence, the refracted waves show their presence, mainly, at intermediate frequencies.

3) Partition of incident energy at anisotropic porous ocean bottom is quite sensitive to the choice of propagation plane to which incident wave is confined. In a given propagation plane, the presence of monoclinic symmetry in anisotropy does not have much effect on the energy partition but the presence of orthotropic symmetry does affect all the reflected and refracted waves.

4) The reflected $P$ wave and refracted $q P_{2}$ wave are nearly insensitive to the presence of anelasticity in the porous frame. Effect of anelasticity is, however, observed on the other three refracted waves but only for incidence beyond their critical angles in respective elastic version.

5) Increase in tortuosity of pores strengthens the reflected $P$ wave but weaken the refracted $q P_{2}$ wave. Other refracted wave may weaken a little with the increase of tortuosity but only for post-critical incidence.
6) Pore-opening at the ocean bottom matters more for propagation at high frequency. The reflected wave gets stronger with the increase of surface flow impedance for partially opened bottom pores. However, for fixed surface flow impedance, the reflected wave weakens with the opening of pores at ocean bottom.

7) The diagnostic (i.e., $q P_{2}$ ) wave in porous bed is very much sensitive to the surface flow impedance as well as the opening of surface pores. This wave is strengthens manifold with the opening of surface pores but weakens a lot with the increase of surface flow impedance.

\section{Appendix A}

The coefficient matrices in Christoffel equations are $\mathbf{A}=\mathbf{A}^{(2)}-\frac{1}{R} \boldsymbol{\Psi}^{(4)} ; \quad \mathbf{B}=\mathbf{A}^{(1)}-\frac{1}{R} \boldsymbol{\Psi}^{(3)} ; \quad \mathbf{C}=\mathbf{A}^{(0)}-$ $\frac{1}{R} \boldsymbol{\Psi}^{(2)} ; \quad \mathbf{D}^{\prime}=-\frac{1}{R} \boldsymbol{\Psi}^{(1)} ; \quad \mathbf{E}^{\prime}=-\frac{1}{R} \boldsymbol{\Psi}^{(0)} ;$

where, for $c_{i j}$ denoting two-indexed notations of elastic tensor $c_{i j k l}$,

$\mathbf{A}^{(2)}=\left\{c_{56}, c_{45}, c_{35} ; c_{45}, c_{44}, c_{34} ; c_{35}, c_{34}, c_{33}\right\}$;

$A_{11}^{(1)}=2\left(c_{15} p_{1}+c_{56} p_{2}\right)$

$A_{12}^{(1)}=A_{21}^{(1)}=\left(c_{14}+c_{56}\right) p_{1}+\left(c_{25}+c_{46}\right) p_{2}$;

$A_{13}^{(1)}=A_{31}^{(1)}=\left(c_{13}+c_{55}\right) p_{1}+\left(c_{36}+c_{45}\right) p_{2}$;

$A_{22}^{(1)}=2\left(c_{46} p_{1}+c_{24} p_{2}\right)$;

$A_{23}^{(1)}=A_{32}^{(1)}=\left(c_{36}+c_{45}\right) p_{1}+\left(c_{23}+c_{44}\right) p_{2}$;

$A_{33}^{(1)}=2\left(c_{35} p_{1}+c_{34} p_{2}\right)$;

$A_{11}^{(0)}=c_{11} p_{1}^{2}+c_{66} p_{2}^{2}+2 c_{16} p_{1} p_{2}-\rho ;$

$A_{12}^{(0)}=A_{21}^{(0)}=c_{16} p_{1}^{2}+c_{26} p_{2}^{2}+\left(c_{12}+c_{66}\right) p_{1} p_{2}$;

$A_{13}^{(0)}=A_{31}^{(0)}=c_{15} p_{1}^{2}+c_{46} p_{2}^{2}+\left(c_{14}+c_{56}\right) p_{1} p_{2}$;

$A_{22}^{(0)}=c_{66} p_{1}^{2}+c_{22} p_{2}^{2}+2 c_{26} p_{1} p_{2}-\rho ;$

$A_{23}^{(0)}=A_{32}^{(0)}=c_{56} p_{1}^{2}+c_{24} p_{2}^{2}+\left(c_{25}+c_{46}\right) p_{1} p_{2}$;

$A_{33}^{(0)}=c_{55} p_{1}^{2}+c_{44} p_{2}^{2}+2 c_{45} p_{1} p_{2}-\rho$; and

$\Psi^{(4)}=\boldsymbol{\Phi}^{(4)} / a_{2}$;

$\Psi^{(3)}=\left(\boldsymbol{\Phi}^{(3)}-a_{1} \Psi^{(4)}\right) / a_{2}$;

$\boldsymbol{\Psi}^{(2)}=\left(\boldsymbol{\Phi}^{(2)}-a_{1} \boldsymbol{\Psi}^{(3)}-a_{0} \boldsymbol{\Psi}^{(4)}\right) / a_{2}$;

$\boldsymbol{\Psi}^{(1)}=\left(\boldsymbol{\Phi}^{(1)}-a_{1} \boldsymbol{\Psi}^{(2)}-a_{0} \boldsymbol{\Psi}^{(3)}\right) / a_{2}$;

$\boldsymbol{\Psi}^{(0)}=\left(\boldsymbol{\Phi}^{(0)}-a_{0} \boldsymbol{\Psi}^{(2)}\right) / a_{2}, \quad$ with

$\Phi_{i j}^{(4)}=m_{i 3} \Gamma_{3 j}^{(2)}$;

$\Phi_{i j}^{(3)}=m_{i 3}\left(p_{k} \Gamma_{k j}^{(2)}+\Gamma_{3 j}^{(1)}\right)+X_{i} \Gamma_{3 j}^{(2)}$;

$\Phi_{i j}^{(2)}=m_{i 3}\left(p_{k} \Gamma_{k j}^{(1)}+\Gamma_{3 j}^{(0)}\right)+X_{i}\left(p_{k} \Gamma_{k j}^{(2)}+\Gamma_{3 j}^{(1)}\right)-\rho_{\mathrm{f}} \Gamma_{i j}^{(2)} ;$

$\Phi_{i j}^{(1)}=m_{i 3} p_{k} \Gamma_{k j}^{(0)}+X_{i}\left(p_{k} \Gamma_{k j}^{(1)}+\Gamma_{3 j}^{(0)}\right)-\rho_{\mathrm{f}} \Gamma_{i j}^{(1)} ;$

$\Phi_{i j}^{(0)}=X_{i} p_{k} \Gamma_{k j}^{(0)}-\rho_{\mathrm{f}} \Gamma_{i j}^{(0)}$,

where, $X_{j}=m_{j 1} p_{1}+m_{j 2} p_{2},(j=1,2,3)$, and

$\Gamma_{11}^{(2)}=\rho_{\mathrm{f}} \chi_{22}+m_{13} X_{123}$;

$\Gamma_{11}^{(1)}=-2 \rho_{\mathrm{f}} \chi_{23} p_{2}+m_{13}\left(X_{23} p_{1}+X_{132} p_{2}\right)+X_{1} X_{123}$;

$\Gamma_{11}^{(0)}=X_{1}\left(X_{13} p_{1}+X_{132} p_{2}\right)-\rho_{\mathrm{f}}\left(X_{23}-\chi_{33} p_{2}^{2}\right)$;

$\Gamma_{12}^{(2)}=-\rho_{\mathrm{f}} \chi_{12}+m_{23} X_{123}$;

$\Gamma_{12}^{(1)}=\rho_{\mathrm{f}}\left(\chi_{23} p_{1}+\chi_{13} p_{2}\right)+m_{23}\left(X_{23} p_{1}+X_{132} p_{2}\right)+X_{2} X_{123}$;

$\Gamma_{12}^{(0)}=X_{2}\left(X_{23} p_{1}+X_{132} p_{2}\right)-\rho_{\mathrm{f}}\left(X_{132}+\chi_{33} p_{1} p_{2}\right)$;

$\Gamma_{13}^{(2)}=m_{33} X_{123}$;

$\Gamma_{13}^{(1)}=\rho_{\mathrm{f}}\left(\chi_{12} p_{2}-\chi_{22} p_{1}\right)+m_{33}\left(X_{23} p_{1}+X_{132} p_{2}\right)+X_{3} X_{123}$;

$\Gamma_{13}^{(0)}=X_{3}\left(X_{23} p_{1}+X_{132} p_{2}\right)-\rho_{\mathrm{f}}\left(X_{123}+\chi_{13} p_{2}^{2}-\chi_{23} p_{1} p_{2}\right)$;

$\Gamma_{21}^{(2)}=-\rho_{\mathrm{f}} \chi_{12}+m_{13} X_{213}$

$\Gamma_{21}^{(1)}=\rho_{\mathrm{f}}\left(\chi_{13} p_{2}+\chi_{23} p_{1}\right)+m_{13}\left(X_{13} p_{2}+X_{132} p_{1}\right)+X_{1} X_{213}$;

$\Gamma_{21}^{(0)}=X_{1}\left(X_{13} p_{2}+X_{132} p_{1}\right)-\rho_{\mathrm{f}}\left(X_{132}+\chi_{33} p_{1} p_{2}\right)$; 
$\Gamma_{22}^{(2)}=\rho_{\mathrm{f}} \chi_{11}+m_{23} X_{213}$;

$\Gamma_{22}^{(1)}=-2 \rho_{\mathrm{f}} \chi_{13} p_{1}+m_{23}\left(X_{13} p_{2}+X_{132} p_{1}\right)+X_{2} X_{213}$;

$\Gamma_{22}^{(0)}=X_{2}\left(X_{13} p_{2}+X_{132} p_{1}\right)-\rho_{\mathrm{f}}\left(X_{13}-\chi_{33} p_{1}^{2}\right)$;

$\Gamma_{23}^{(2)}=m_{33} X_{213}$;

$\Gamma_{23}^{(1)}=\rho_{\mathrm{f}}\left(\chi_{12} p_{1}-\chi_{11} p_{2}\right)+m_{33}\left(X_{132} p_{1}+X_{13} p_{2}\right)+X_{3} X_{213}$;

$\Gamma_{23}^{(0)}=X_{3}\left(X_{13} p_{2}+X_{132} p_{1}\right)-\rho_{\mathrm{f}}\left(X_{213}+\chi_{23} p_{1}^{2}-\chi_{13} p_{1} p_{2}\right)$;

$\Gamma_{31}^{(2)}=m_{13} X_{12}$;

$\Gamma_{31}^{(1)}=\rho_{\mathrm{f}}\left(\chi_{12} p_{2}-\chi_{22} p_{1}\right)+m_{13}\left(X_{123} p_{1}+X_{213} p_{2}\right)+X_{1} X_{12}$;

$\Gamma_{31}^{(0)}=X_{1}\left(X_{123} p_{1}+X_{213} p_{2}\right)-\rho_{\mathrm{f}}\left(X_{123}+\chi_{13} p_{2}^{2}-\chi_{23} p_{1} p_{2}\right)$;

$\Gamma_{32}^{(2)}=m_{23} X_{12}$;

$\Gamma_{32}^{(1)}=\rho_{\mathrm{f}}\left(\chi_{12} p_{1}-\chi_{11} p_{2}\right)+m_{23}\left(X_{123} p_{1}+X_{213} p_{2}\right)+X_{2} X_{12}$;

$\Gamma_{32}^{(0)}=X_{2}\left(X_{123} p_{1}+X_{213} p_{2}\right)-\rho_{\mathrm{f}}\left(X_{213}+\chi_{23} p_{1}^{2}-\chi_{13} p_{1} p_{2}\right)$;

$\Gamma_{33}^{(2)}=m_{33} X_{12}$;

$\Gamma_{33}^{(1)}=m_{33}\left(X_{123} p_{1}+X_{213} p_{2}\right)+X_{3} X_{12}$;

$\Gamma_{33}^{(0)}=X_{3}\left(X_{123} p_{1}+X_{213} p_{2}\right)-\rho_{\mathrm{f}}\left(X_{12}+2 \chi_{12} p_{1} p_{2}-\chi_{11} p_{2}^{2}-\right.$ $\left.\chi_{22} p_{1}^{2}\right)$.

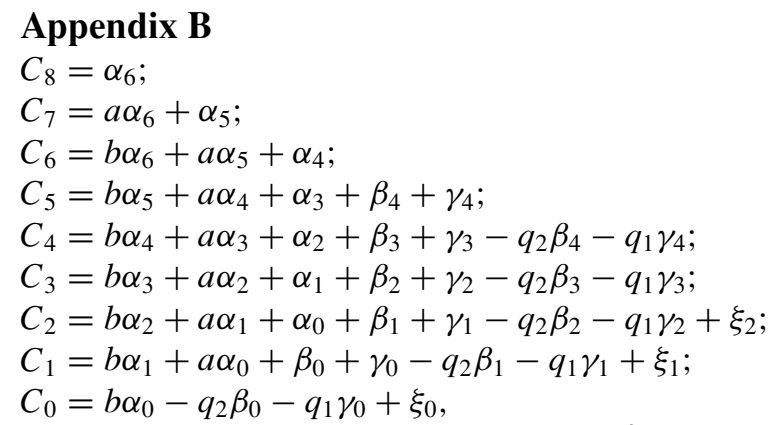

where, $q_{1}$ and $q_{2}$ are two roots of equation $q^{2}+a q+b=0$. Define $|X Y Z|$ as the determinant of a matrix obtained by selecting first row from matrix $\mathbf{X}$, second row from matrix $\mathbf{Y}$ and third row from matrix $\mathbf{Z}$. Then, the various coefficients are expresses as follows.

$\alpha_{6}=|A A A|$

$\alpha_{5}=|A A B|+|A B A|+|B A A|$

$\alpha_{4}=|A A C|+|A C A|+|C A A|+|A B B|+|B A B|+|B B A|$

$\alpha_{3}=|A B C|+|A C B|+|B A C|+|B C A|+|C A B|+$ $|C B A|+|B B B|$;

$\alpha_{2}=|A C C|+|C A C|+|C C A|+|B B C|+|B C B|+|C B B|$;

$\alpha_{1}=|B C C|+|C B C|+|C C B|$;

$\alpha_{0}=|C C C|$;

$\beta_{4}=|A A D|+|A D A|+|D A A|$

$\beta_{3}=|A B D|+|A D B|+|B A D|+|B D A|+|D A B|+$

$|D B A|$;

$\beta_{2}=|A C D|+|A D C|+|C A D|+|C D A|+|D A C|+$ $|D C A|+|B B D|+|B D B|+|D B B|$;

$\beta_{1}=|B C D|+|B D C|+|C B D|+|C D B|+|D B C|+$ $|D C B|$;

$\beta_{0}=|C C D|+|C D C|+|D C C|$;

$\gamma_{4}=|A A E|+|A E A|+|E A A|$;

$\gamma_{3}=|A B E|+|A E B|+|B A E|+|B E A|+|E A B|+|E B A|$;

$\gamma_{2}=|A C E|+|A E C|+|C A E|+|C E A|+|E A C|+$

$|E C A|+|B B E|+|B E B|+|E B B|$;

$\gamma_{1}=|B C E|+|B E C|+|C B E|+|C E B|+|E B C|+|E C B|$;

$\gamma_{0}=|C C E|+|C E C|+|E C C|$;

$\xi_{2}=|A D E|+|A E D|+|D A E|+|D E A|+|E A D|+$

$|E D A|$;

$\xi_{1}=|B D E|+|B E D|+|D B E|+|D E B|+|E B D|+$

$|E D B|$;
$\xi_{0}=|C D E|+|C E D|+|D C E|+|D E C|+|E C D|+$ $|E D C|$.

With the matrices $\mathbf{A}, \mathbf{B}, \mathbf{C}, \mathbf{D}^{\prime}, \mathbf{E}^{\prime}$ as defined in Appendix $\mathrm{A}, \mathbf{D}=\left(\mathbf{E}^{\prime}+\mathbf{D}^{\prime} q_{1}\right) /\left(q_{1}-q_{2}\right)$ and $\mathbf{E}=\left(\mathbf{E}^{\prime}+\right.$ $\left.\mathbf{D}^{\prime} q_{2}\right) /\left(q_{2}-q_{1}\right)$. In case of repeated roots for $q^{2}+a q+b=$ 0 , the matrices $\mathbf{D}=\mathbf{D}^{\prime} ; \quad \mathbf{E}=\mathbf{E}^{\prime}-0.5 a \mathbf{D}$. The changes in $C_{j}$ are as follows.

$C_{5}=b \alpha_{5}+a \alpha_{4}+\alpha_{3}+\beta_{4}$

$C_{4}=b \alpha_{4}+a \alpha_{3}+\alpha_{2}+\beta_{3}+\gamma_{4}+0.5 a \beta_{4}$

$C_{3}=b \alpha_{3}+a \alpha_{2}+\alpha_{1}+\beta_{2}+\gamma_{3}+0.5 a \beta_{3}$;

$C_{2}=b \alpha_{2}+a \alpha_{1}+\alpha_{0}+\beta_{1}+\gamma_{2}+0.5 a \beta_{2}$

$C_{1}=b \alpha_{1}+a \alpha_{0}+\beta_{0}+\gamma_{1}+0.5 a \beta_{1}$

$C_{0}=b \alpha_{0}+0.5 a \beta_{0}+\gamma_{0}$.

\section{References}

Ainslie, M. A. and P. W. Burns, Energy-conserving reflection and transmission coefficients for a solid-solid boundary, J. Acoust. Soc. Am., 98 , 2836-2840, 1995.

Albert, D. G., A comparison between wave propagation in water-saturated and air-saturated porous materials, J. Appl. Phys., 73, 28-36, 1993.

Badiey, M., L. Jaya, and A. H.-D. Cheng, Propagator matrix for plane wave reflection from inhomogeneous anisotropic poroelastic seafloor, J. Comput. Acoust., 2, 11-27, 1994.

Biot, M. A., The theory of propagation of elastic waves in a fluid-saturated porous solid, I. Low-frequency range, II. Higher frequency range, $J$. Acoust. Soc. Am., 28, 168-191, 1956.

Biot, M. A., Mechanics of deformation and acoustic propagation in porous media, J. Appl. Phys., 33, 1482-1498, 1962a.

Biot, M. A., Generalized theory of acoustic propagation in porous dissipative media, J. Acoust. Soc. Am., 34, 1254-1264, 1962b.

Borcherdt, R. D., Reflection and refraction of type-II $S$ waves in elastic and inelastic media, Bull. Seism. Soc. Am., 67, 43-67, 1977.

Buckingham, M. J., Theory of acoustic attenuation, dispersion and pulse propagation in unconsolidated granular materials including marine sediments, J. Acoust. Soc. Am., 102, 2579-2596, 1997.

Buckingham, M. J., Theory of compressional and shear waves in fluid like marine sediments, J. Acoust. Soc. Am., 103, 288-299, 1998.

Buckingham, M. J., Theory of compressional and transverse wave propagation in consolidated porous media, J. Acoust. Soc. Am., 106, 575-587, 1999.

Buckingham, M. J., Wave propagation, stress relaxation, and grain-tograin shearing in saturated, unconsolidated marine sediments, J. Acoust. Soc. Am., 108, 2796-2815, 2000.

Carcione, J. M., Wave Fields in Real Media: Wave Propagation in Anisotropic, Anelastic and Porous Media, Pergamon, Amsterdam, 2001.

Chotiras, N. P., D. E. Smith, and J. N. Piper, Refraction and scattering into a sandy ocean sediment in $30-40 \mathrm{kHz}$ band, IEEE J. Oceanic Engng. 27, 362-375, 2007.

Collins, M. D., J. F. Lingevitch, and W. L. Siegmann, Wave propagation in poro-acoustic media, Wave Motion, 25, 265-272, 1997.

Crampin, S., Suggestions for a consistent terminology for seismic anisotropy, Geophys. Prospect., 37, 753-770, 1989.

Crampin, S., The fracture criticality of crustal rocks, Geophys. J. Int., 118 , 428-438, 1994.

de la Cruz, V., J. Hube, and T. J. T. Spanos, Reflection and transmission of seismic waves at the boundaries of porous media, Wave Motion, 16, 323-338, 1992.

Denneman, A. I. M., G. G. Drijkoningen, D. M. J. Smeulders, and K. Wapenar, Reflection and transmission of waves at a fluid/porous medium interface, Geophys., 67, 282-291, 2002.

Deresiewicz, H. and R. Skalak, On uniqueness in dynamic Poroelasticity, Bull. Seismol. Soc. Am., 53, 793-799, 1963.

Gurevich, B., Elastic properties of saturated porous rocks with aligned fractures, J. Appl. Geophys., 54, 203-218, 2003.

Johnson, D. L., J. Koplik, and R. Dashen, Theory of dynamic permeability and tortuosity in fluid-saturated porous media, J. Fluid Mech., 176, 379402, 1987.

Kohler, W. E., A one-dimensional randomly stratified model of ocean sediments, Wave Motion, 10, 421-441, 1988.

Kuo, E. Y. T., Acoustic wave scattering from two solid boundaries at the ocean bottom: Reflection loss, IEEE J. Oceanic Eng., 17, 159-170, 
1992.

Lin, C.-H., V. M. Lee, and M. D. Trifunac, The reflection of plane waves in a poroelastic half-space saturated with inviscid fluid, Soil Dyn. Earthquake, 25, 206-223, 2006.

Liu, J. Y., C. F. Huang, and S. W. Shyue, Effect of seabed properties on acoustic wave fields in a seismo-acoustic ocean waveguide, Ocean Eng., 28, 1437-1459, 2001.

Potel, C. and F. J. de Belleval, Propagation in an anisotropic periodically multilayered medium, J. Acoust. Soc. Am., 93, 2669-2677, 1993.

Rasolofosaon, P. N. J. and B. E. Zinszner, Comparison between permeability anisotropy and elasticity anisotropy of reservoir rocks, Geophys., 67, 230-240, 2002.

Schmitt, D. P., Acoustic multipole logging in transversely isotropic poroelastic formations, J. Acoust. Soc. Am., 86, 2397-2421, 1989.

Sharma, M. D., Three-dimensional wave propagation in a general anisotropic poroelastic medium: phase velocity, group velocity and polarisation, Geophys. J. Int., 156, 329-344, 2004a.

Sharma, M. D., 3-D wave propagation in a general anisotropic poroelastic medium: reflection and refraction at an interface with fluid, Geophys. $J$. Int., 157, 947-958, 2004b.

Sharma, M. D., Propagation of inhomogeneous plane waves in dissipative anisotropic poroelastic solids, Geophys. J. Int., 163, 981-990, 2005.

Sharma, M. D., Propagation of harmonic plane waves in a general anisotropic porous solid, Geophys. J. Int., 2007 (in press).

Sharma, M. D. and M. L. Gogna, Wave propagation in anisotropic liquidsaturated porous solids, J. Acoust. Soc. Am., 89, 1068-1073, 1991.
Stoll, R. D., Acoustic waves in saturated sediment, in Physics of sound in Marine Sediments, edited by L. Hampton, 19-39, Plenum, New York, 1974.

Stoll, R. D., Acoustic waves in Ocean sediments, Geophys., 42, 715-725, 1977.

Stoll, R. D., Experimental studies of attenuation in sediments, J. Acoust. Soc. Am., 66, 1152-1160, 1979.

Stoll, R. D., Theoretical aspects of sound transmissions in sediments, $J$. Acoust. Soc. Am., 68, 1341-1350, 1980.

Stoll, R. D. and G. M. Bryan, Wave attenuation in saturated sediments, $J$. Acoust. Soc. Am., 47, 1440-1447, 1970.

Stoll, R. D. and T.-K. Kan, Reflection of acoustic waves at a watersediment interface, J. Acoust. Soc. Am., 70, 149-156, 1981.

Vashishth, A. K. and P. Khurana, Rayleigh modes in anisotropic, heterogeneous poroelastic layers, J. Seismol., 9, 431-448, 2005.

Yang, J., Importance of flow condition on seismic waves at a saturated porous solid boundary, J. Sound Vib., 221, 391-413, 1999.

Yamamoto, T., Acoustic propagation in the ocean with a poro-elastic bottom, J. Acoust. Soc. Am., 73, 1587-1596, 1983.

Yamamoto, T., Acoustic scattering in the ocean from velocity and density fluctuations in the sediments, J. Acoust. Soc. Am., 99, 866-879, 1996.

A. K. Vashishth (e-mail: anil_vashishth@yahoo.co.in) and M. D. Sharma 